\title{
THE REISSNER-SAGOCI PROBLEM $\dagger$
}

\author{
by IAN N. SNEDDON
}

(Received 23 April, 1965)

1. The statical Reissner-Sagoci problem $[1,2,3]$ is that of determining the components of stress and displacement in the interior of the semi-infinite homogeneous isotropic elastic solid $z \geqq 0$ when a circular area $(0 \leqq \rho \leqq a, z=0)$ of the boundary surface is forced to rotate through an angle $\alpha$ about an axis which is normal to the undeformed plane surface of the medium. It is easily shown that, if we use cylindrical coordinates $(\rho, \phi, z)$, the displacement vector has only one non-vanishing component $u_{\phi}(\rho, z)$, and the stress tensor has only two non-vanishing components, $\sigma_{\rho \phi}(\rho, z)$ and $\sigma_{\phi z}(\rho, z)$. The stress-strain relations reduce to the two simple equations

$$
\sigma_{\rho \phi}=\mu \rho \frac{\partial}{\partial \rho}\left(\rho^{-1} u_{\phi}\right), \quad \sigma_{\phi z}=\mu \frac{\partial u_{\phi}}{\partial z},
$$

where $\mu$ is the shear modulus of the material. From these equations, it follows immediately that the equilibrium equation

$$
\frac{\partial \sigma_{\rho \phi}}{\partial \rho}+\frac{\partial \sigma_{\phi z}}{\partial z}+\frac{2 \sigma_{\rho \phi}}{\rho}=0
$$

is satisfied provided that the function $u_{\phi}(\rho, z)$ is a solution of the partial differential equation

$$
\frac{\partial^{2} u_{\phi}}{\partial \rho^{2}}+\frac{1}{\rho} \frac{\partial u_{\phi}}{\partial \rho}-\frac{u_{\phi}}{\rho^{2}}+\frac{\partial^{2} u_{\phi}}{\partial z^{2}}=0
$$

The boundary conditions can be written in the form

$$
\begin{aligned}
u_{\phi}(\rho, 0) & =f(\rho) & & (0 \leqq \rho \leqq a), \\
\sigma_{\phi z}(\rho, 0) & =0 & & (\rho>a),
\end{aligned}
$$

where, in the case in which we are most interested, $f(\rho)=\alpha \rho$. We also assume that, as $r \rightarrow \infty$, $u_{\phi}, \sigma_{\rho \phi}$ and $\sigma_{\phi z}$ all tend to zero.

A solution of this boundary value problem by the use of a Hankel transform to reduce the problem to that of solving a pair of dual integral equations was given in [4]. The final form of this solution is complicated because the solution of the dual integral equation is taken in the rather complicated form due to Titchmarsh [5].

In $\S 2$ of the present paper the solution of the Reissner-Sagoci problem is again derived in the form of the Hankel transform of order 1 of a function which is determined in terms of $f(\rho)$ by the same pair of dual integral equations, but, instead of using Titchmarsh's solution, we make use of an elementary solution closely related to the one given in [6] for the corresponding mixed boundary value problem in potential theory. This enables us to derive a

† This work is supported by a grant, AFOSR-444-64, under the sponsorship of the Joint Services Advisory Group through AFOSR, ARO and ONR at North Carolina State University. 
simple equation for the torque $T$ in terms of the arbitrary function $f(\rho)$. As an example of the use of the formulae derived in $\S 2$ the problem in which $f(\rho)=\alpha \rho$ is considered in $\S 3$.

Finally in $\S 4$ the solution is adapted to provide a solution of the problem of the torsional distortion of a semi-infinite circular cylinder $0 \leqq \rho \leqq b, z \geqq 0$ when its plane face is subjected to the boundary conditions (1.3) and (1.4) and its curved surface is rigidly clamped so that we have the boundary condition

$$
u_{\phi}(b, z)=0 \quad(z \geqq 0) .
$$

2. It is easily shown that the displacement $u_{\phi}(\rho, z)$ can be written in terms of the Hankel transform of order 1 of a function involving an arbitrary factor $A(\xi)$, i.e., that we can write

$$
u_{\phi}(\rho, z)=\mathscr{H}_{1}\left[\xi^{-1} A(\xi) e^{-\xi z} ; \xi \rightarrow \rho\right],
$$

where the operator $\mathscr{H}_{v}$ is defined by the equation

$$
\mathscr{H}_{v}[\psi(\xi, z) ; \xi \rightarrow \rho]=\int_{0}^{\infty} \xi \psi(\xi, z) J_{v}(\xi \rho) d \xi .
$$

If we substitute this expression into the second of the equations (1.1), we find that

$$
\sigma_{\phi z}(\rho, z)=-\mu \mathscr{H}_{1}\left[A(\xi) e^{-\xi z} ; \xi \rightarrow \rho\right] ;
$$

and if we substitute it into the first of the same pair of equations and make use of the recurrence relation

$$
\rho \frac{\partial}{\partial \rho}\left\{\rho^{-1} J_{1}(\xi \rho)\right\}=-\xi J_{2}(\xi \rho),
$$

we see that the second component of stress is given by the equation

$$
\sigma_{\rho \phi}(\rho, z)=-\mu \mathscr{H}_{2}\left[A(\xi) e^{-\xi z} ; \xi \rightarrow \rho\right] .
$$

From equations (2.1), (2.2) we see immediately that the boundary conditions (1.3), (1.4) are equivalent to the pair of dual integral equations

$$
\begin{aligned}
& \mathscr{H}_{1}\left[\xi^{-1} A(\xi) ; \rho\right]=f(\rho) \quad(0 \leqq \rho \leqq a), \\
& \mathscr{H}_{1}[A(\xi) ; \rho]=0 \quad(\rho>a) .
\end{aligned}
$$

Expressing $A(\xi)$ in terms of an unknown function $g(t)$ through the equation

we find that

$$
A(\xi)=\int_{0}^{a} g(t) \sin (\xi t) d t, \quad \text { where } g(0)=0,
$$

$$
\xi A(\xi)=\int_{0}^{a} g^{\prime}(t) \cos (\xi t) d t-g(a) \cos (\xi a),
$$

and making use of the relation

$$
\int_{0}^{\infty} \cos (\xi t) J_{1}(\xi \rho) d \xi=\frac{1}{\rho}-\frac{t H(t-\rho)}{\rho \sqrt{ }\left(t^{2}-\rho^{2}\right)}
$$


we see that equation (2.5) is automatically satisfied whatever the form of $g(t)$. Similarly, if we make use of the integral

$$
\int_{0}^{\infty} J_{1}(\xi \rho) \sin (\xi t) d \xi=t \rho^{-1}\left(\rho^{2}-t^{2}\right)^{-\frac{1}{2}} H(\rho-t),
$$

we see that equation (2.4) is satisfied if we take $g(t)$ to be the solution of the integral equation

$$
\int_{0}^{\rho} \frac{t g(t) d t}{\sqrt{\left(\rho^{2}-t^{2}\right)}}=\rho f(\rho) \quad(0 \leqq \rho \leqq a) .
$$

This is an equation of Abel type, which is easily solved to give

$$
g(t)=\frac{2}{\pi} \int_{0}^{t} \frac{d}{d \rho}\{\rho f(\rho)\} \frac{d \rho}{\sqrt{\left(t^{2}-\rho^{2}\right)}} \quad(0 \leqq t \leqq a) .
$$

The solution to the problem is therefore given by equations (2.1), (2.6a) and (2.9).

The torque $T$ which must be applied to produce the prescribed boundary conditions is given by the equation

$$
T=-2 \pi \int_{0}^{a} \rho^{2} \sigma_{\phi z}(\rho, 0) d \rho .
$$

If we substitute from equation (2.2) into this equation and make use of the result

$$
\int_{0}^{a} \rho^{2} J_{1}(\xi \rho) d \rho=\frac{a^{2}}{\xi} J_{2}(a \xi)
$$

we find that

$$
T=2 \pi \mu a^{2} \mathscr{H}_{2}\left[\xi^{-1} A(\xi) ; a\right]
$$

and recalling that

$$
\int_{0}^{\infty} J_{2}(a \xi) \sin (\xi t) d \xi=\frac{2 t}{a^{2}} \quad(0 \leqq t \leqq a),
$$

we obtain the equation

$$
T=4 \pi \mu \int_{0}^{a} t g(t) d t .
$$

We now note that we can write equation (2.9) in the form

$$
g(t)=\frac{2}{\pi t} \frac{d}{d t} \int_{0}^{t} \frac{\rho^{2} f(\rho) d \rho}{\sqrt{\left(t^{2}-\rho^{2}\right)}},
$$

from which we deduce that

$$
T=8 \mu \int_{0}^{a} \frac{\rho^{2} f(\rho) d \rho}{\sqrt{\left(a^{2}-\rho^{2}\right)}}
$$


We can express the other quantities of physical interest in terms of $g(t)$. For example, using equations (2.1), (2.6a) and (2.8), we find that

$$
u_{\phi}(\rho, 0)=\frac{1}{\rho} \int_{0}^{a} \frac{\operatorname{tg}(t) d t}{\sqrt{\left(\rho^{2}-t^{2}\right)}} \quad(\rho>a) .
$$

Similarly, from equations (2.2), (2.6b) and (2.7), we deduce that

$$
\sigma_{\phi z}(\rho, 0)=\frac{\mu}{\rho} \int_{p}^{a} \frac{t g^{\prime}(t) d t}{\sqrt{\left(t^{2}-\rho^{2}\right)}}-\frac{\mu a}{\rho} \cdot \frac{g(a)}{\sqrt{\left(a^{2}-\rho^{2}\right)}} \quad(0 \leqq \rho<a) .
$$

Also, if we substitute from equation (2.6b) into equation (2.3) and make use of the result

$$
\int_{0}^{\infty} \cos (\xi t) J_{2}(\rho \xi) d \xi=\frac{\left(\rho^{2}-2 t^{2}\right) H(\rho-t)}{\rho^{2} \sqrt{ }\left(\rho^{2}-t^{2}\right)},
$$

we find that

$$
\sigma_{\rho \phi}(\rho, 0)=-\frac{\mu}{\rho^{2}}\left\{\int_{0}^{m} \frac{\left(\rho^{2}-2 t^{2}\right) g^{\prime}(t) d t}{\sqrt{ }\left(\rho^{2}-t^{2}\right)}-\frac{\rho^{2}-2 a^{2}}{\sqrt{ }\left(\rho^{2}-a^{2}\right)} g(a) H(\rho-a)\right\},
$$

where $m=\min (\rho, a)$.

Finally, we note that if we substitute from equation (2.6a) into equation (2.1) and make use of the result

$$
\int_{0}^{\infty} e^{-p \xi} J_{1}(\rho \xi) d \xi=\frac{1}{\rho}\left[1-\frac{p}{\sqrt{ }\left(p^{2}+\rho^{2}\right)}\right](\operatorname{Re} p>0)
$$

we may write

$$
u_{\phi}(\rho, z)=\frac{1}{2 i \rho} \int_{-a}^{a} \frac{(z+i t) g(t) d t}{\sqrt{\left[\rho^{2}+(z+i t)^{2}\right]}},
$$

where $g(t)$ is an odd function of $t$ defined for $t>0$ by equation (2.9). This is the form of solution used by Green and Zerna. (See p. 173 of [7]).

3. As an example of the use of these formulae we consider the special case in which $f(\rho)=\alpha \rho$. It is then easily shown from equation (2.9) that $g(t)=4 \alpha t / \pi$ and hence from equation (2.6a) that

$$
A(\xi)=\frac{4 \alpha}{\pi \xi^{2}}(\sin \xi a-\xi a \cos \xi a)
$$

From equation (2.10) we deduce immediately that

$$
T=\frac{16}{3} \mu \alpha a^{3} \text {. }
$$


Similarly from equations (2.12), (2.13) and (2.14) we have, respectively, the relations

$$
\begin{aligned}
& u_{\phi}(\rho, 0)=\frac{2 \alpha}{\pi}\left[\rho \sin ^{-1}\left(\frac{a}{\rho}\right)-\frac{a}{\rho} \sqrt{ }\left(a^{2}-\rho^{2}\right)\right] \quad(\rho>a), \\
& \sigma_{\phi z}(\rho, 0)=-\frac{4 \alpha \mu \rho}{\pi \sqrt{ }\left(a^{2}-\rho^{2}\right)}(0 \leqq \rho<a), \\
& \sigma_{\rho \phi}(\rho, 0)=-\frac{4 \alpha \mu}{\pi \rho^{2}}\left[m \sqrt{ }\left[\rho^{2}-m^{2}\right)-\frac{a\left(\rho^{2}-2 a^{2}\right)}{\sqrt{\left(\rho^{2}-a^{2}\right)}} H(\rho-a)\right],
\end{aligned}
$$

where as before $m=\min (\rho, a)$.

The displacement and the components of stress at an interior point of the elastic halfspace can be calculated by substituting from equation (3.1) into equations (2.1), (2.2), (2.3) and making use of known results concerning the integrals involved [8].

4. We shall now consider the problem of determining the distribution of stress in the interior of a very long circular cylinder of radius $b$ of homogeneous isotropic material when a circular area $0 \leqq \rho \leqq a$ of its flat end $z=0$ is forced to rotate through an angle $\alpha$ about the axis of the cylinder. We suppose also that the curved surface $\rho=b, z \geqq 0$ of the cylinder is fixed. We therefore have the boundary conditions (1.3), (1.4) and (1.5). We shall find it more convenient to use the equivalent relation

$$
\mathscr{F}_{c}\left[u_{\phi}(b, z) ; z \rightarrow \xi\right]=0
$$

instead of (1.5), where $\mathscr{F}_{c}$ denotes the operator of the Fourier cosine transform defined by the equation

$$
\mathscr{F}_{c}[f(\rho, z) ; z \rightarrow \xi]=\sqrt{\frac{2}{\pi}} \int_{0}^{\infty} f(\rho, z) \cos (\xi z) d z .
$$

To the simple solution (2.1), we add a second function which is also a solution of equation (1.2):

$$
u_{\phi}(\rho, z)=\mathscr{H}_{1}\left[\zeta^{-1} A(\zeta) e^{-\xi z} ; \zeta \rightarrow \rho\right]+\mathscr{F}_{c}\left[\xi^{-1} B(\xi) I_{1}(\xi \rho) ; \xi \rightarrow z\right] .
$$

From this displacement we derive the stress components

$$
\begin{aligned}
& \sigma_{\phi z}(\rho, z)=-\mu \mathscr{H}_{1}\left[A(\zeta) e^{-\xi z} ; \zeta \rightarrow \rho\right]-\mu_{F_{s}}\left[B(\xi) I_{1}(\xi \rho) ; \xi \rightarrow z\right], \\
& \sigma_{\rho \phi}(\rho, z)=-\mu \mathscr{H}_{2}\left[A(\zeta) e^{-\xi z}, \zeta \rightarrow \rho\right]+\mu \mathscr{F}_{c}\left[B(\xi) I_{2}(\xi \rho) ; \xi \rightarrow z\right],
\end{aligned}
$$

where $\mathscr{F}_{s}$ is the operator of the Fourier sine transform, so that the boundary conditions (1.3), (1.4) reduce to the pair of dual integral equations

$$
\begin{array}{rlrl}
\mathscr{H}_{1}\left[\zeta^{-1} A(\zeta) ; \rho\right]+\sqrt{\frac{2}{\pi}} \int_{0}^{\infty} \xi^{-1} B(\xi) I_{1}(\xi \rho) d \xi & =f(\rho) & & (0 \leqq \rho \leqq a), \\
\mathscr{H}_{1}[A(\zeta) ; \rho] & =0 & (\rho>a) .
\end{array}
$$


If we again take the representation $(2.6 \mathrm{a}, \mathrm{b})$ for $A$, we see that equation (4.6) is identically satisfied, and noting that

$$
\begin{aligned}
\frac{2}{\pi} \int_{0}^{t} \frac{d \rho}{\sqrt{\left(t^{2}-\rho^{2}\right)}} \frac{d}{d \rho} \sqrt{\frac{2}{\pi}} \int_{0}^{\infty} \xi^{-1} B(\xi) \rho I_{1}(\xi \rho) d \xi & =\left(\frac{2}{\pi}\right)^{3 / 2} \int_{0}^{\infty} B(\xi) d \xi \int_{0}^{t} \frac{\rho I_{0}(\xi \rho) d \rho}{\sqrt{\left(t^{2}-\rho^{2}\right)}} \\
& =\left(\frac{2}{\pi}\right)^{3 / 2} \int_{0}^{\infty} \xi^{-1} B(\xi) \sinh (\xi t) d \xi
\end{aligned}
$$

we find on comparing with equation (2.9) that the equation (4.5) is equivalent to

$$
g(t)+\left(\frac{2}{\pi}\right)^{3 / 2} \int_{0}^{\infty} \xi^{-1} B(\xi) \sinh (\xi t) d \xi=h(t)
$$

where

$$
h(t)=\frac{2}{\pi} \int_{0}^{t} \frac{d}{d \rho}\{\rho f(\rho)\} \frac{d \rho}{\sqrt{\left(t^{2}-\rho^{2}\right)}}
$$

Since

$$
\mathscr{F}_{c}\left[e^{-\xi z} ; z \rightarrow \xi\right]=\sqrt{\frac{2}{\pi}} \frac{\zeta}{\xi^{2}+\zeta^{2}}, \quad \mathscr{H}_{1}\left[\frac{\sin \zeta t}{\xi^{2}+\zeta^{2}} ; \zeta \rightarrow \rho\right]=\sinh (\xi t) K_{1}(\xi \rho) \quad(\rho>t),
$$

we see from equation (4.2) that the boundary condition (4.1) is equivalent to

$$
\xi^{-1} B(\xi) I_{1}(b \xi)+\sqrt{\frac{2}{\pi}} K_{1}(b \xi) \int_{0}^{a} g(\tau) \sinh (\xi \tau) d \tau=0 .
$$

If we eliminate $B(\xi)$ between this equation and equation (4.7), we see that $g(t)$ is the solution of the Fredholm integral equation of the second kind

$$
g(t)-\int_{0}^{a} g(\tau) K(t, \tau) d \tau=h(t) \quad(0 \leqq t \leqq a),
$$

where the free term is given by equation (4.8), and the kernel by the equation

$$
K(t, \tau)=L(t+\tau)-L(|t-\tau|)
$$

with

$$
L(w)=\frac{2}{\pi^{2}} \int_{0}^{\infty} \frac{K_{1}(\xi b)}{I_{1}(\xi b)}[\cosh (\xi w)-1] d \xi .
$$

We note also that the torque is still given by equation (2.10). In the case in which $f(\rho)=\alpha \rho, h(t)=4 \alpha t / \pi$, so that, if we write

$$
g(t)=2 a \alpha \phi(t / a)
$$

K 
we find that $\phi(t)$ is the solution of the Fredholm equation

$$
\phi(t)-\int_{0}^{1} \phi(u) K_{1}(t, u) d u=\frac{2}{\pi} t \quad(0 \leqq t \leqq 1),
$$

whose kernel is defined by the equations

$$
\begin{gathered}
K_{1}(t, u)=L_{1}\left(\frac{t+u}{c}\right)-L_{1}\left(\frac{|t-u|}{c}\right), \\
L_{1}(w)=\frac{2}{\pi^{2} c} \int_{0}^{\infty} \frac{K_{1}(\eta)}{I_{1}(\eta)}[\cosh (w \eta)-1] d \eta
\end{gathered}
$$

with $c=b / a$.

Also in terms of $\phi(t)$, we find that the torque is given by the equation

When $b \gg a, T$ takes the value

$$
T=8 \pi \mu \alpha a^{3} \int_{0}^{1} t \phi(t) d t .
$$

$$
T_{\infty}=\frac{16}{3} \mu \alpha a^{3}
$$

so that we have the relation

$$
\frac{T}{T_{\infty}}=\frac{3 \pi}{2} \int_{0}^{1} t \phi(t) d t
$$

The integral equation (4.10), with kernel defined by the pair of equations (4.11) has been solved numerically by Sneddon and Tait [9] and the quantity $T / T_{\infty}$ calculated. The values obtained are shown in Table 1.

TABLE 1

\begin{tabular}{|c|l|l|l|l|l|l|l|}
\hline$b / a$ & 1.05 & 1.10 & 1.20 & 1.30 & 1.6667 & 2.5 & 5.0 \\
$T / T_{\infty}$ & 1.9704 & 1.6649 & 1.3670 & 1.2725 & 1.0994 & 1.0205 & 1.0027 \\
\hline
\end{tabular}

In the same paper an iterative solution (for small values of $a / b$ ) of equation (4.10) was derived. This yields the formula

$$
\frac{T}{T_{\infty}}=1+0.3382 \frac{a^{3}}{b^{3}}+0.0815 \frac{a^{5}}{b^{5}}+0.1144 \frac{a^{6}}{b^{6}}+0.0125 \frac{a^{7}}{b^{7}}+O\left(a^{8} / b^{8}\right) .
$$

The solution of this problem can be derived in an entirely different way. If we assume a displacement of the form

$$
u_{\phi}(\rho, z)=\sum_{n=1}^{\infty} \alpha_{n}^{-1} u_{n} e^{-a_{n} z} J_{1}\left(\alpha_{n} \rho\right)
$$


then the boundary condition (1.5) will be satisfied if we take the constants $\alpha_{n}$ to be the positive zeros of $J_{1}\left(\alpha_{n} b\right)$ written in ascending order of magnitude, and the boundary conditions (1.3), (1.4) will be satisfied if we choose the constants $u_{n}$ to be the solution of the dual series relations

$$
\begin{gathered}
\sum_{n=1}^{\infty} \alpha_{n}^{-1} u_{n} J_{1}\left(\alpha_{n} \rho\right)=f(\rho) \quad(0 \leqq \rho \leqq a), \\
\sum_{n=1}^{\infty} u_{n} J_{1}\left(\alpha_{n} \rho\right)=0 \quad(a<\rho<b) .
\end{gathered}
$$

Dual series relations of this type have been discussed by Cooke and Tranter [10] and more recently by Sneddon and Srivastav [11]. The solution in [10] is reduced to that of an infinite set of linear algebraic equations for the constants $u_{n}$, while the solution in [11] consists in showing that if the function on the left side of equation (4.16) is represented in the closed interval $[0, a]$ by an integral of a form equivalent to that in equation (2.13) involving a function $g(t)$, then $g(t)$ is the solution of the integral equation (4.9).

It is interesting to note that when we attempt to solve the Reissner-Sagoci problem for a cylinder whose curved surface is stress-free, i.e., when the condition (1.5) is replaced by the condition $\sigma_{\rho \phi}(b, z)=0, z \geqq 0$, the procedure given above can be repeated purely formally; but we find that the integral defining the kernel in the integral equation (4.9) is

$$
\frac{4}{\pi^{2}} \int_{0}^{\infty} \frac{K_{2}(\xi b)}{I_{2}(\xi b)} \sinh (u \xi) \sinh (\tau \xi) d \xi
$$

and this is divergent since the integrand is $O\left(\xi^{-2}\right)$ as $\xi \rightarrow 0$. On the other hand, by assuming a displacement of the form (4.14) but with the Fourier-Bessel series on the right replaced by a Dini series, we can derive a pair of dual series relations which can be solved by a method due to Srivastav [12]. The details of the calculation are given in a subsequent paper [13].

\section{REFERENCES}

1. E. Reissner, Freie und erzwungene Torsionschwingungen des elastischen Halbraumes, Ing.-Arch, 8 (1937), 229-245.

2. E. Reissner and H. F. Sagoci, Forced torsional oscillations of an elastic half-space, I, J. Appl. Phys. 15 (1944), 652-654. $655-662$.

3. H. F. Sagoci, Forced torsional oscillations of an elastic half-space, II, J. Appl. Phys. 15 (1944),

4. I. N. Sneddon, Note on a boundary value problem of Reissner and Sagoci, J. Appl. Phys. 18 (1947), 130-132.

5. E. C. Titchmarsh, Introduction to the theory of Fourier integrals (Clarendon Press, Oxford, 1937), 334-339.

6. I. N. Sneddon, The elementary solution of dual integral equations, Proc. Glasgow Math. Assoc. 4 (1960), 108-110.

7. A. E. Green and W. Zerna, Theoretical elasticity (Clarendon Press, Oxford, 1954).

8. D. L. George, Numerical values of some integrals involving Bessel functions, Proc. Edinburgh Math. Soc. (2) 13 (1962), 87-113. 
9. I. N. Sneddon and R. J. Tait, The effect of a penny-shaped crack on the distribution of stress in a long circular cylinder, Internat. J. Eng. Sci. 1 (1963), 391-409.

10. J. C. Cooke and C. J. Tranter, Dual Fourier-Bessel series, Quart. J. Mech. and Appl. Math. 12 (1959), 379-385.

11. I. N. Sneddon and R. P. Srivastav, Dual series relations, I, Proc. Roy. Soc. Edinburgh A 66 (1963), 150-160.

12. R. P. Srivastav, Dual series relations, II, Proc. Roy. Soc. Edinburgh A 66 (1963), 161-172.

13. I. N. Sneddon, R. P. Srivastav and S. C. Mathur, The Reissner-Sagoci problem for a long cylinder of finite radius. (To appear in Quart.J. Mech. and Appl. Math.)

\section{UNIVERSITY OF GLASGOW}

GlasGow, W. 2 